\section{ORIGINAL RESEARCH}

\author{
A. Bizzi \\ G. Castelli \\ M. Bugiani \\ P.B. Barker \\ E.H. Herskovits \\ U. Danesi \\ A. Erbetta \\ I. Moroni \\ L. Farina \\ G. Uziel
}

\title{
Classification of Childhood White Matter Disorders Using Proton MR Spectroscopic Imaging
}

\begin{abstract}
BACKGROUND AND PURPOSE: Childhood white matter disorders often show similar MR imaging signal-intensity changes, despite different underlying pathophysiologies. The purpose of this study was to determine if proton MR spectroscopic imaging ( $\left.{ }^{1} \mathrm{H}-\mathrm{MRSI}\right)$ may help identify tissue pathophysiology in patients with leukoencephalopathies.
\end{abstract}

\begin{abstract}
MATERIALS AND METHODS: Seventy patients (mean age, 6; range, 0.66-17 years) were prospectively examined by ${ }^{1} \mathrm{H}-\mathrm{MRSI}$; a diagnosis of leukoencephalopathy due to known genetic defects leading to lack of formation, breakdown of myelin, or loss of white matter tissue attenuation (rarefaction) was made in 47 patients. The diagnosis remained undefined (UL) in 23 patients. Patients with definite diagnoses were assigned (on the basis of known pathophysiology) to 3 groups corresponding to hypomyelination, white matter rarefaction, and demyelination. Choline (Cho), creatine $(\mathrm{Cr})$, and $\mathrm{N}$ acetylaspartate (NAA) signals from 6 white matter regions and their intra- and intervoxel (relative to gray matter) ratios were measured. Analysis of variance was performed by diagnosis and by pathophysiology group. Stepwise linear discriminant analysis was performed to construct a model to predict pathophysiology on the basis of ${ }^{1} \mathrm{H}-\mathrm{MRSI}$, and was applied to the UL group.
\end{abstract}

RESULTS: Analysis of variance by diagnosis showed 3 main metabolic patterns. Analysis of variance by pathophysiology showed significant differences for Cho/NAA $(P<.001)$, $\mathrm{Cho} / \mathrm{Cr}(P<.004)$, and $\mathrm{NAA} / \mathrm{Cr}(P<.002)$. Accuracy of the linear discriminant analysis model was $75 \%$, with $\mathrm{Cho} / \mathrm{Cr}$ and $\mathrm{NAA} / \mathrm{Cr}$ being the best parameters for classification. On the basis of the linear discriminant analysis model, $61 \%$ of the subjects in the UL group were classified as hypomyelinating.

CONCLUSION: ${ }^{1} \mathrm{H}-\mathrm{MRSI}$ provides information on tissue pathophysiology and may, therefore, be a valuable tool in the evaluation of patients with leukoencephalopathies.

L eukoencephalopathies constitute a large group of inherited and acquired diseases that predominantly involve the cerebral white matter. ${ }^{1-3}$ The enzyme defects leading to dysfunction and breakdown of myelin have been identified in an increasing number of genetic leukoencephalopathies that have their onset in infancy or childhood. ${ }^{3}$ Nevertheless, approximately $30 \%$ of leukoencephalopathies remain of unknown etiology, despite extensive clinical, imaging, and laboratory investigations.

Three main pathophysiologic mechanisms may affect myelin and lead to white matter abnormalities. "Hypomyelination" is defined as a deficit in the formation of myelin, whereas "demyelination" is defined as loss or destruction of previously formed myelin; "rarefaction" identifies a process that leads to reduction of overall white matter tissue attenuation and the eventual replacement of white matter by CSF. Progressive rarefaction may be the result of myelin vacuolation in megalencephalopathy with subcortical cysts (MLC) ${ }^{4}$ and in a subtype

Received January 28, 2008; accepted after revision February 24.

From the Departments of Neuroradiology (A.B., G.C., U.D., A.E., L.F.) and Child Neurology (M.B., I.M., G.U.), Fondazione IRCCS Istituto Neurologico "Carlo Besta," Milan, Italy; Department of Radiology (E.H.H.), University of Pennsylvania, Philadelphia, Pa; and Russell H Morgan Department of Radiology and Radiological Science (P.B.B.), Johns Hopkins University, Baltimore, Md.

This work was supported in part by the Pierfranco and Luisa Mariani Foundation and National Institutes of Health P41RR15241.

Please address correspondence to Alberto Bizzi, MD, Dept of Neuroradiology, Fondazione IRCCS Istituto Neurologico "Carlo Besta," Via Celoria, 11, 20133 Milan, Italy; e-mail: alberto_bizzi@fastwebnet.it

Indicates article with supplemental on-line tables and figures.

DOI 10.3174/ajnr.A1106 of mitochondrial disorders with predominant white matter signal-intensity abnormalities, ${ }^{5}$ or of cystic degeneration in leukoencephalopathy with vanishing white matter (LVWM). ${ }^{4}$

MR imaging is highly sensitive and plays a prominent role in the diagnostic evaluation of these patients. The imaging hallmark of leukoencephalopathies is symmetric signal-intensity abnormalities in white matter. ${ }^{3}$ However, the specificity of MR imaging is not particularly high, and leukoencephalopathies of different pathophysiologies may show similar signalintensity changes on T1- and T2-weighted MR images.

Proton MR spectroscopy $\left({ }^{1} \mathrm{H}-\mathrm{MRS}\right)$ measures in vivo concentrations of a few brain metabolites that may be affected in leukoencephalopathies. ${ }^{6}$ Multivoxel ${ }^{1} \mathrm{H}-\mathrm{MRSI}^{7,8}$ allows the simultaneous acquisition of multiple spectra from gray and white matter regions, usually at higher spatial resolution than single-voxel ${ }^{1} \mathrm{H}$-MRSI. With recent advances in MR imaging technology, ${ }^{1} \mathrm{H}$-MRSI has become more widely available, and it can be included easily in the same session as conventional MR imaging. However, the role of ${ }^{1} \mathrm{H}-\mathrm{MRSI}$ in the diagnostic work-up of patients with leukoencephalopathies is not yet well defined, because few studies have used ${ }^{1} \mathrm{H}$-MRSI in large numbers of patients with leukoencephalopathies of different pathophysiologies. ${ }^{9,10}$

In this study, the hypotheses that different leukoencephalopathies sharing the same underlying pathophysiology exhibit similar metabolic patterns, and that identification of these patterns allows classification of leukoencephalopathies based on ${ }^{1} \mathrm{H}$-MRSI findings, were tested. Therefore, according to their definitive diagnoses, patients were assigned to 1 of the 3 pathophysiology groups: hypomyelination, demyelination, 
and rarefaction; and ${ }^{1} \mathrm{H}-\mathrm{MRSI}$ metabolite ratios were compared among groups. A second aim of the study was to construct a model by using linear discriminant analysis to accurately separate patients into the 3 pathophysiology groups. Finally, as an example of a clinical application, the linear discriminant analysis model was used to assign a pathophysiology group to the 23 patients with undefined leukoencephalopathy (UL). Classification of leukoencephalopathies into 3 main groups may help to narrow the differential diagnosis and thereby direct biochemical and genetic testing.

\section{Materials and Methods}

\section{Patients}

In this prospective study, conventional MR imaging and MR spectroscopy were performed for consecutive children admitted to the Carlo Besta Neurologic Institute during an 8-year period (1998-2005). This study was performed with the informed consent of the patients' parents. Three board-certified child neurologists (M.B., I.M., G.U.) performed neurologic examinations on these patients. Seventy patients $(\mathrm{M} / \mathrm{F}$ ratio $=$ 39:31; mean age, 6 years and 1 month; range, 8 months to 17 years) met the following inclusion criteria: 1) symmetric white matter signal-intensity abnormalities on MR images; 2) predominant or exclusive white matter involvement on MR images; and 3) appropriate clinical, molecular, and genetic investigations necessary to diagnose defined hereditary leukoencephalopathies.

A definite diagnosis was made in 47 children. They were assigned to a pathophysiology group: 7 patients had a hypomyelinating disorder (due to the following gene mutations: 3 with PLP, 2 with GJA12, 1 with LAMA2, and 1 with DRCTNNB1A); 12 had a disease leading to rarefaction of white matter ( 4 with genetically proven LVWM, 2 with an $M L C 1$ mutation, and 6 with mitochondrial disorders identified by a defect of the respiratory chain complex: I in 1, II in 3, and IV in 2 patients); 25 had a demyelinating disorder (4 with Alexander disease, 8 with adrenoleukodystrophy, 6 with globoid cell leukodystrophy, and 7 with metachromatic leukodystrophy, all genetically or enzymatically proven). Two patients were diagnosed with Canavan disease and 1, with L-2-OH glutaric aciduria. The diagnosis remained UL in 23 patients (on-line Tables 1 and 2). A tentative diagnosis based on clinical examination, other diagnostic tests including conventional MR imaging, and long-term follow-up was made by 3 board-certified pediatric neurologists (M.B., I.M., G.U.).

\section{MR Imaging and MR Spectroscopic Imaging}

All examinations were performed at 1.5T (ACSII Gyroscan; Philips, Best, the Netherlands). The imaging protocol included sagittal and axial T1-, proton-spin density and T2-weighted spin-echo, and coronal and/or axial FLAIR images. ${ }^{1} \mathrm{H}$-MRSI was performed during the same session in all patients with a single-section multivoxel $2 \mathrm{D}$ phaseencoded point-resolved spectroscopy sequence $(\mathrm{TR}=1500 \mathrm{~ms}, \mathrm{TE}=$ $136 \mathrm{~ms})$ with a nominal planar resolution of $10 \times 10 \mathrm{~mm}(\mathrm{FOV}=$ $160 \times 160 \mathrm{~mm}^{2}$; matrix $\left.=16 \times 16\right)$ and $15-\mathrm{mm}$ section thickness. At $\mathrm{TE}=136 \mathrm{~ms}$, there is inversion of the lactate signal intensity which helps distinguish it from (noninverted) lipid signals. In all patients, the volume of interest was positioned at the level of the centrum semiovale above the roof of the lateral ventricles. Water suppression was performed with a selective inversion pulse after automatic shimming and gradient tuning. Scan acquisition time was 8 minutes.

\section{Quantitative Analysis}

${ }^{1} \mathrm{H}$-MRSI raw data were transferred to a Sun Workstation (Sun Microsystems, Palo Alto, Calif), reconstructed and processed by using "csx2" software, developed at Johns Hopkins University. ${ }^{11}$ After baseline correction by using a cubic-spline routine, peak areas of choline (Cho), creatine (Cr), and $N$-acetylaspartate (NAA) were estimated by numeric integration. Metabolite signals were measured from single spectra in the anterior, central, and posterior regions of white matter (common regions of involvement) and parasagittal cortical gray matter of both cerebral hemispheres. Spectra were integrated independently by 2 spectroscopists, and the average of the measurements was taken. White matter intravoxel (Cho/NAA, Cho/Cr, and NAA/Cr) and normalized (nCho, nCr, nNAA) ratios were calculated as the average of the 6 white matter voxels. The spectrum in the parasagittal parietal gray matter was used to calculate the normalized ratios. Spectra in the cortical gray matter were relatively spared in all types of leukoencephalopathies included in this study. However, there are caveats of using the cortical gray matter because some types of leukoencephalopathies may affect the gray matter more than others. Abnormal accumulation of lactate was noted in each case; however, lactate signal intensity was not quantified.

\section{Statistical Analyses}

Three levels of statistical analysis were performed by using the program Statistical Package for the Social Sciences Version 9.0 for Windows (SPSS, Chicago, Ill). First, analysis of variance for each of the 6 metabolite measures was performed according to diagnosis. Diseases with only 1 case (hyccin deficiency, congenital muscular dystrophy, and $\mathrm{L} 2-\mathrm{OH}$ glutaric aciduria) were excluded from this analysis. Tukey Honest Significant Difference post hoc test was used to estimate statistical significance.

Second, analysis of variance was performed with 44 patients classified into 3 groups according to underlying tissue pathophysiology. The following disorders were considered to belong to group 1 (hypomyelination): Pelizaeus-Merzbacher disease, Pelizaeus-Merzbacherlike disease, hyccin deficiency, and congenital muscular dystrophy. LVWM, mitochondrial disorders, and MLC were classified in group 2 as diseases involving rarefaction of the white matter. Metachromatic leukodystrophy, globoid cell leukodystrophy, Alexander disease, and adrenoleukodystrophy were classified in group 3 (demyelination). The 3 patients diagnosed with organic aciduria were not included in this analysis because Canavan disease has a relatively unique biochemical defect leading to marked NAA accumulation, ${ }^{12} \mathrm{~L}-2-\mathrm{OH}$ glutaric aciduria has a different pathophysiology, and only 1 patient with this disease was recruited in this study. On adding age as a covariate to the analysis of variance of patients with definite diagnosis, we found no interaction among age and any of the 6 measured metabolites or ratios and no interactions with either diagnosis or pathophysiology. Therefore, 1-way analysis of variance was performed, rather than analysis of covariance, to assess differing metabolic states across diagnostic and pathophysiologic groups.

Third, stepwise linear discriminant analysis was performed to select metabolite ratios in constructing a model that accurately separates patients into the 3 pathophysiology groups; the goal was to build a tool that can classify new patients before a diagnosis is made. The diagnostic accuracy of this model was measured on the training set of 44 patients with definite diagnosis by using leave-one-out cross-validation. The model was then used to assign a pathophysiology group to the 23 patients with UL. Agreement between the classifier and the 
presumed pathophysiology group based on tentative diagnosis was determined in UL patients.

\section{Results}

The results of Cho/NAA, Cho/Cr, and NAA/Cr measurement in the white matter of 47 patients with defined diagnoses are illustrated in on-line Fig 1A; 3 different patterns of metabolite intravoxel ratios were observed. The hypomyelinating disorders (Pelizaeus-Merzbacher disease, Pelizaeus-Merzbacherlike disease, hyccin deficiency, and congenital muscular dystrophy) manifested Cho/NAA, Cho/Cr, and NAA/Cr ratios that were similar to those observed in healthy children older than 2 years (ie, Cho/NAA approximately 0.6 , Cho/Cr approximately 1.0 , and NAA/Cr $>1.5^{13}$ ). In leukoencephalopathies with white matter rarefaction (LVWM, mitochondrial disorders, MLC), the Cho/NAA, Cho/Cr, and NAA/Cr were approximately 1.0, whereas demyelinating diseases (Alexander disease, adrenoleukodystrophy, globoid cell leukodystrophy, and metachromatic leukodystrophy) showed elevated Cho/ $\mathrm{NAA}(>1.5)$ and $\mathrm{Cho} / \mathrm{Cr}(>1.4)$ and reduced $\mathrm{NAA} / \mathrm{Cr}$ $(<1.1)$. In on-line Fig $1 B$, the white matter intravoxel ratios of these patients are grouped by pathophysiology; there are clear metabolite-ratio differences among the 3 groups.

Consistent with these observations, post hoc tests for analysis of variance by disease revealed significant differences for Cho/NAA and NAA/Cr (on-line Table 3). Cho/NAA appears useful in differentiating metachromatic leukodystrophy from Pelizaeus-Merzbacher disease, Pelizaeus-Merzbacher-like disease, LVWM, mitochondrial disease, and Canavan disease. NAA/Cr appears useful primarily in differentiating PelizaeusMerzbacher disease and Canavan disease from mitochondrial disorders, MLC, Alexander disease, adrenoleukodystrophy, globoid cell leukodystrophy, and metachromatic leukodystrophy, in addition to differentiating Pelizaeus-Merzbacher-like disease and LVWM from a few of the other disorders.

Post hoc tests for analysis of variance by pathophysiology showed the following differences in: Cho/NAA between hypomyelination and demyelination $(P<.001)$ and between rarefaction and demyelination $(P<.001)$; Cho/Cr between hypomyelination and demyelination $(P=.004)$ and between rarefaction and demyelination $(P=.002)$; and NAA/Cr between hypomyelination and rarefaction $(P=.002)$, between hypomyelination and demyelination $(P<.001)$, and between rarefaction and demyelination $(P=.010)$.

White-matter nCho, $\mathrm{nCr}$, and nNAA ratios are plotted in on-line Fig 2. nCho tended to be higher in demyelinating diseases, but this trend did not reach statistical significance. Similarly, there were no statistically significant differences in $\mathrm{nCr}$ or nNAA among groups, though diseases with white matter rarefaction or demyelination had lower nNAA than hypomyelinating diseases.

Illustrative MR imaging and ${ }^{1} \mathrm{H}$-MRSI data for representative patients of the 3 pathophysiology groups are shown in the following figures: hypomyelination (Fig 1), white matter rarefaction (Fig 2), demyelination (Fig 3), and Canavan disease (Fig 4). Visual inspection of the metabolic images and selected white matter spectra revealed large differences in the relative amplitudes of the NAA and Cho signals among patients, consistent with the results summarized in on-line Figs 1 and 2.
Stepwise linear discriminant analysis between pathophysiology groups resulted in 2 functions:

$$
\begin{aligned}
& f_{1}=-0.443 \times \frac{C h o}{C r}+0.835 \times \frac{N A A}{C r} \\
& f_{2}=0.909 \times \frac{C h o}{C r}+0.569 \times \frac{N A A}{C r} .
\end{aligned}
$$

The values of the 2 canonical discriminant functions are shown in on-line Fig 3, including the centroids and the discriminant lines separating the 3 groups. The results of leaveone-out cross-validation of discriminant analysis by pathophysiology are shown in on-line Tables 1 and 2. Overall, 33 of the 44 cases $(75 \%)$ were correctly classified, which differs significantly from results expected by chance $\left(\chi^{2}, P<1.5 \times\right.$ $\left.10^{-6}\right)$. Two of 7 patients with hypomyelination were misclassified as having rarefaction, and 3 of 12 patients with rarefaction were misclassified: 2 as having hypomyelination and 1 as having demyelination. Six of 25 patients with demyelinating were incorrectly classified: 1 as having hypomyelination and 5 as having rarefaction.

Lactate was elevated in 11 of 12 patients with rarefaction and in 20 of 25 patients with demyelination, whereas it was absent in all patients with hypomyelination (on-line Tables 1 and 2).

The pathophysiology group for the 23 UL cases was determined by using the ${ }^{1} \mathrm{H}$-MRSI model previously described (online Tables 1 and 2). Agreement between the classifier and the pathophysiology group based on tentative diagnosis was found in 14 of the $23(61 \%)$ patients (on-line Tables 1 and 2). Agreement was higher (73\%) for hypomyelinating disorders.

\section{Discussion}

A major finding of this study is that ${ }^{1} \mathrm{H}$-MRSI is able to predict underlying pathophysiology across a relatively wide spectrum of hereditary leukoencephalopathies with an accuracy of $75 \%$. Three broad metabolic profiles, corresponding to white matter hypomyelination, rarefaction, and demyelination, were consistently identified, suggesting that ${ }^{1} \mathrm{H}$-MRSI provides insight into the pathophysiology underlying the highly sensitive but nonspecific signal-intensity abnormalities.

Cho/NAA, Cho/Cr, and NAA/Cr were all significantly different for the demyelinating group compared with either the hypomyelination or the rarefaction group, whereas only NAA/Cr was significantly different between the rarefaction and hypomyelination groups. Linear discriminant analysis based on $\mathrm{Cho} / \mathrm{Cr}$ and NAA/Cr resulted in significant separation of patients from all 3 groups. Intravoxel white matter ratios were found to separate the 3 groups better than ratios normalized to gray matter, due in part to variable gray matter involvement among different leukoencephalopathies. Therefore, intravoxel ratios should be given more importance than intervoxel ratios when evaluating patients with leukoencephalopathies.

Overall, 75\% of hereditary leukoencephalopathies were correctly classified by discriminant analysis into the 3 pathophysiology groups. There are several possible explanations for the $25 \%$ misclassified patients, including the effects of age, timing of the ${ }^{1} \mathrm{H}$-MRSI study relative to the stage of disease progression, spatial heterogeneity of metabolic abnormalities, 

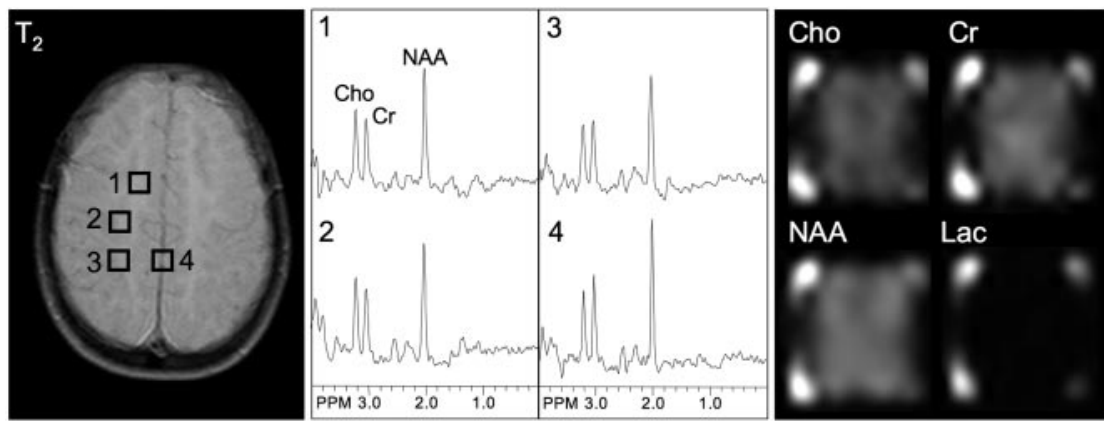

Fig 1. T2-weighted MR, selected spectra, and metabolic images of Cho, Cr, NAA, and lactate (Lac) in a typical case of hypomyelination (Pelizaeus-Merzbacher disease). Spectral patterns from both white and gray matter show near-normal profiles, with a large NAA signal intensity and near-equal Cho and $\mathrm{Cr}$. Bright signal at the corners of the metabolic images arises from residual lipid signals from the scalp. PPM indicates parts per million.
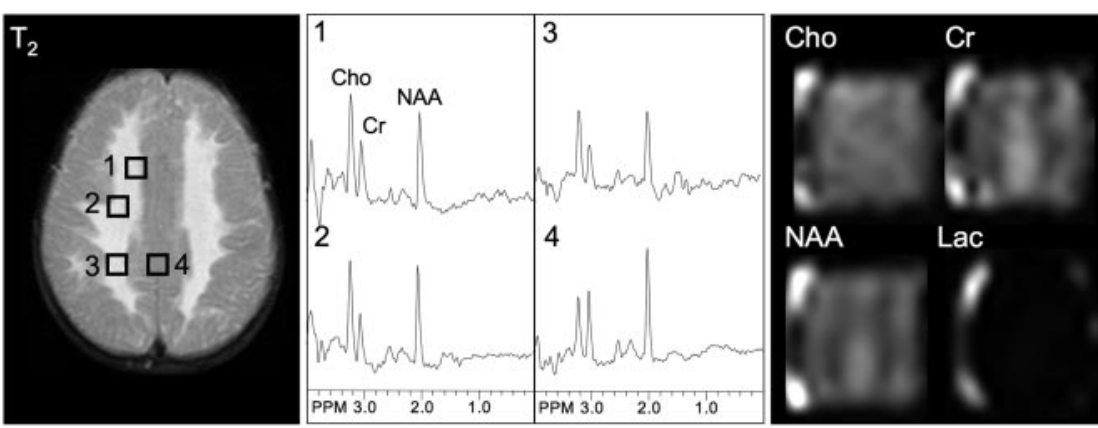

Fig 2. T2-weighted MR, selected spectra, and metabolic images of Cho, Cr, NAA, and lactate (Lac) in a case of rarefaction of white matter (LVWM). Metabolic images clearly show decreased $\mathrm{Cr}$ and NAA in T2-hyperintense white matter regions, whereas Cho is largely unchanged. In white matter voxels, the NAA/Cho ratio is approximately 1:1. This patient was diagnosed with LVWM at a very early stage and is 1 of the 2 patients with LVWM misclassified in group 1. PPM indicates parts per million.
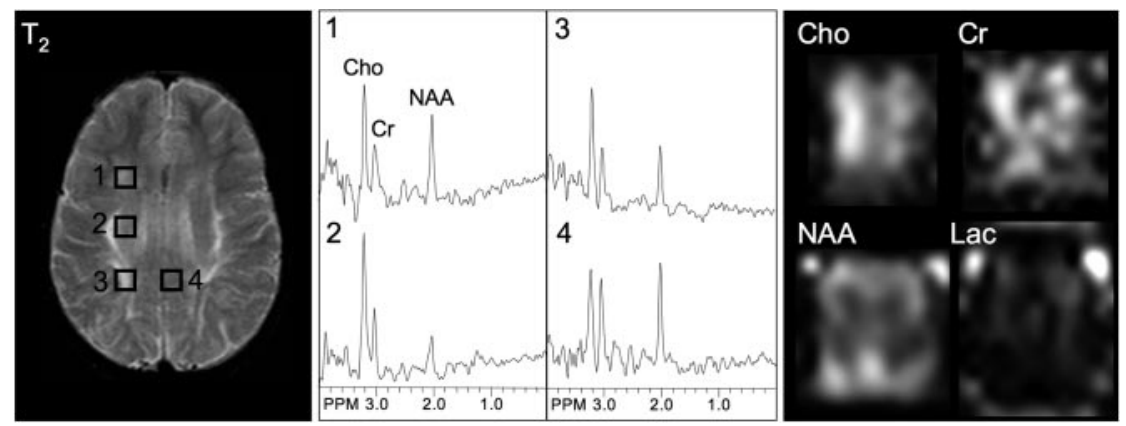

Fig 3. T2-weighted MR, selected spectra, and metabolic images of $\mathrm{Cho}, \mathrm{Cr}$, NAA, and lactate (Lac) in a typical demyelination case (metachromatic leukodystrophy). T2-hyperintense white matter regions are characterized by increased Cho and decreased NAA. The gray matter spectrum (voxel 4) also shows a somewhat decreased NAA/Cho ratio. PPM indicates parts per million.
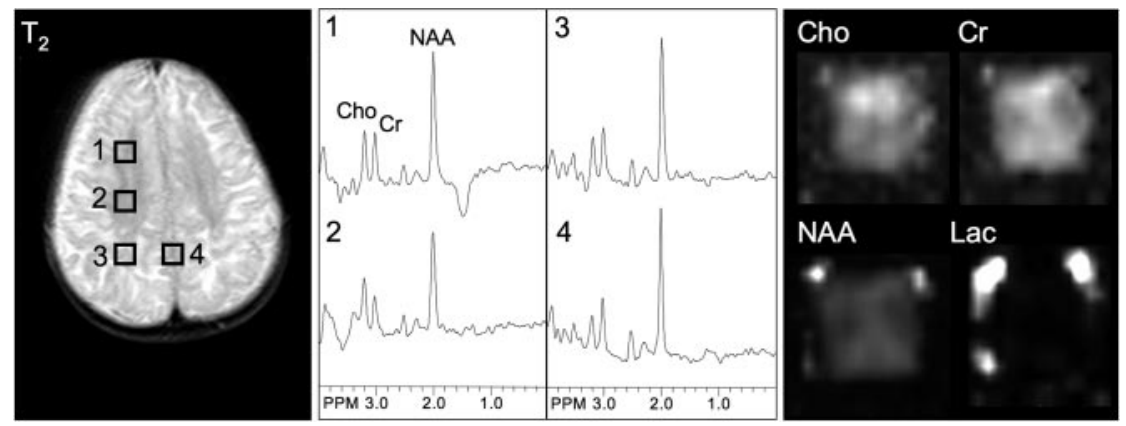

Fig 4. T2-weighted MR, selected spectra, and metabolic images of Cho, $\mathrm{Cr}$, NAA, and lactate (Lac) in a typical patient with Canavan disease. NAA is higher than normal in both white and gray matter regions. Note lower (ie, more normal) NAA levels, as seen in voxel 2 and in the NAA metabolic image, in the deeper white matter where there is less signal-intensity abnormality on the T2-weighted MR image. PPM indicates parts per million.

and phenotypic variations of disease expression. These factors are considered in detail below.

The patient age range was quite large in this study, with the youngest child being 8 months of age. Although we could not demonstrate an interaction between metabolite ratios and age as a covariate, metabolite levels change during development, ${ }^{13}$ particularly during the first 2 years of life, and the wide range in this study clearly increased the heterogeneity of the ${ }^{1} \mathrm{H}$-MRSI data. The youngest 2 patients with hypomyelination were misclassified into the rarefaction group. Both patients were younger than 1 year of age, and their $\mathrm{NAA} / \mathrm{Cr}$ ratios were below the average of the hypomyelina- tion group, which led them to be categorized into group 2 . Several studies of normal brain development have shown that NAA/Cr increases rapidly during the first 2 years of life. Therefore, a limitation of this study is that the classifier developed here is probably less accurate for patients younger than 2 years of age.

Two patients with early-stage LVWM had near-normal NAA/Cr ratios and were misclassified as having hypomyelination. However, follow-up ${ }^{1} \mathrm{H}$-MRSI studies showed progressive decrease in NAA/Cr, and these patients may have been correctly classified if follow-up ${ }^{1} \mathrm{H}$-MRSI data had been included in the discriminant analysis. Therefore, patients with rarefaction in the early stage may be incorrectly classified as 
having hypomyelination by ${ }^{1} \mathrm{H}-\mathrm{MRSI}$. The third misclassified patient had a mitochondrial disorder without cavitations on MR imaging and was assigned to group 3.

Timing of the ${ }^{1} \mathrm{H}-\mathrm{MRSI}$ examination and phenotypic variability may also have resulted in misclassification of 3 patients with globoid cell leukodystrophy (also known as Krabbe disease). One patient had late onset of a relatively mild form of globoid cell leukodystrophy. He was classified in group 1; then his clinical status stabilized after hematopoietic stem cell transplantation. ${ }^{1} \mathrm{H}$-MRSI studies confirmed no significant changes of Cho/NAA and NAA/Cr in subsequent years. Clinical records of the 2 other patients with globoid cell leukodystrophy revealed that they had late onset of the disorder and that they were examined when already in the chronic phase; they were misclassified in group 2. Serial studies in patients with progressive leukoencephalopathy (adrenoleukodystrophy, globoid cell leukodystrophy, and metachromatic leukodystrophy) have shown evolution of metabolites with time, with the highest Cho levels seen in the acute phases of demyelination and low levels of all metabolites seen in the chronic phase. ${ }^{14}$ Thus, ${ }^{1} \mathrm{H}-\mathrm{MRSI}$ and clinical history in these patients could be considered to actually be in agreement, suggesting that these 3 patients did not have active demyelination at the time of the ${ }^{1} \mathrm{H}$-MRSI examination.

An important aspect of the current study design was the use of the same anatomic section from the centrum semiovale in all patients. Metabolite signals of spectra from 9 standardized regions of interest were measured and averaged in all subjects. This allowed consistency of data collection and removed the possible confounding factor of normal regional variation in metabolite levels. However, this approach may decrease sensitivity in patients with heterogeneously distributed lesions. For example, in 3 patients with adrenoleukodystrophy, focal T2 signal-intensity abnormalities were limited to the posterior third of the centrum semiovale, with normal MR imaging signal intensity and metabolite ratios in the anterior and middle regions. Although abnormal spectra from normal-appearing white matter have been reported in some leukoencephalopathies, ${ }^{14,15}$ it is possible that a study probing the region of greatest metabolic abnormality would have increased power for separating leukoencephalopathies of different etiologies.

Normal Cho, Cr, and NAA in leukoencephalopathy with hypomyelination suggest that membrane turnover and axonal density in the white matter are relatively normal, despite a permanent deficit in myelin deposition.

Loss of Cho, Cr, and NAA on ${ }^{1} \mathrm{H}-\mathrm{MRSI}$ is related to rarefaction and cystic degeneration in LVWM, cavity formation in specific phenotypic expressions of mitochondrial disorders, or myelin vacuolation in MLC. In this study, therefore, the use of the term "rarefaction" was to underline reduction of cerebral tissue attenuation, a characteristic feature of the 3 leukoencephalopathies included in group 2. It is well known that childhood mitochondrial encephalopathies have a very broad phenotypic expression on conventional MR imaging examination: Approximately $10 \%$ of cases will predominantly involve the white matter ${ }^{5}$ and will form cavitations. Formation of cysts in the frontal white matter may occur also in end stage Alexander disease. However, marked elevation of Cho was the prominent spectroscopic feature in the acute phase of Alexander disease and was found in all 4 patients.
Elevation of Cho associated with NAA loss is the hallmark of leukoencephalopathies with demyelination. High Cho is believed to result from a combination of several factors: the arrival of inflammatory cells in the lesion, accumulation of myelin breakdown products such as glycerophosphocholine and phosphocholine, ${ }^{16}$ and increased membrane turnover that will increase the amount of free Cho. Accumulation of lipids was not seen in any patients, despite the occurrence of myelin breakdown, particularly in the demyelinating group, probably because of the long TE used (136 ms). ${ }^{1} \mathrm{H}$-MRSI is quite sensitive to the detection of mobile lipids at short TEs $(<30 \mathrm{~ms})$. Myelin breakdown occurs mainly in the form of macromolecules that have very short TEs and are not detectable with in vivo ${ }^{1} \mathrm{H}$-MRSI.

One other recently published study has investigated whether single-voxel ${ }^{1} \mathrm{H}$-MRSI, combined with other advanced MR imaging techniques, can discriminate among leukoencephalopathies with different pathophysiologies. ${ }^{17}$ In that study, total $\mathrm{Cr}$ was found to be the best overall discriminant parameter among groups, followed by total choline, magnetization-transfer ratio, myo-inositol, apparent diffusion coefficient, lactate, and total NAA. ${ }^{17}$ Our study is in general agreement, in that it shows that ${ }^{1} \mathrm{H}$-MRSI is a valuable tool in discriminating among pathophysiology groups. Our results, obtained with only $1 \mathrm{MR}$ imaging technique and in a patient population with a wide range of 13 different leukoencephalopathies, underscore the importance of the ${ }^{1} \mathrm{H}$-MRSI examination in the diagnostic evaluation of these patients. Measurement of the average ratios from 6 white matter locations, rather than 1 single voxel, also allowed wider lesion coverage in the current study.

Finally, the discriminant model built by using 44 hereditary leukoencephalopathies was used to assign pathophysiologygroup membership to the 23 UL patients. Lack of diagnosis is a major problem for patients, parents, and physicians; because prognosis cannot be provided, no treatment is available and prenatal diagnosis is not possible. ${ }^{4}$ Sixty-one percent of UL patients were classified on the basis of ${ }^{1} \mathrm{H}$-MRSI into the hypomyelination group. These results suggest that most UL patients (at least in this series) may have a defect of myelin formation rather than a demyelinating disease.

\section{Conclusion}

This study demonstrates that ${ }^{1} \mathrm{H}-\mathrm{MRSI}$ is a valuable tool for identifying underlying pathophysiology in leukoencephalopathies. Metabolite ratios are generally consistent among diseases with similar pathophysiology, leading to 3 main profiles of metabolite signal intensity ratios. Recognition of these metabolic profiles in individual patients has clinical implications because it may help narrow the list of differential diagnoses at presentation and thereby help set priorities for subsequent biochemical or other testing to be performed in reaching a definitive diagnosis.

\section{References}

1. Aubourg P. The leukodystrophies: a window to myelin. Nat Genet 1993;5: 105-06

2. Barkovich A, Lyon G, Ezrard P. Formation, maturation, and disorders of white matter. AJNR Am J Neuroradiol 992;13:447-61

3. van der Knaap MS, Valk J. Magnetic Resonance Myelination and Myelin Disorders. Berlin, Germany: Springer-Verlag; 2005 
4. van der Knaap M, Breiter S, Naidu S. Defining and categorizing leukoencephalopathies of unknown origin: MR imaging approach. Radiology 1999;213:121-33

5. Moroni I, Bugiani M, Bizzi A, et al. Cerebral white matter involvement in children with mitochondrial encephalopathies. Neuropediatrics 2002;33:79-85

6. Barker PB, Horska A. Neuroimaging in leukodystrophies. J Child Neurol 2004; 19:559-70

7. Duyn JH, Gillen J, Sobering G, et al. Multisection proton MR spectroscopic imaging of the brain. Radiology 1993;188:277-82

8. Golay X, Gillen J, van Zijl PC, et al. Scan time reduction in proton magnetic resonance spectroscopic imaging of the human brain. Magn Reson Med 2002;47:384-87

9. Bizzi A, Bugiani M, Danesi U. MR spectroscopy of inborn errors of metabolism. In: Gillard JH, Waldman AD, Barker PB, eds. Clinical MR Neuroimaging Diffusion, Perfusion and Spectroscopy. Cambridge, UK: Cambridge University Press; 2005:779-802

10. Bizzi A, Danesi U. Proton MR spectroscopy as a diagnostic tool in leukoencephalopathies. In: Uziel G, Taroni F, eds. Hereditary Leukoencephalopathies and Demyelinating Neuropathies in Children. Montrouge, France: John Libbey Eurotext; 2004;65-78
11. Soher B, van Zijl P, Duyn J, et al. Quantitative proton spectroscopic imaging of the human brain. Magn Reson Med 1996;35:356-63

12. Matalon R, Michals K, Sebesta D, et al. Aspartoacylase deficiency and N-acetylaspartic aciduria in patients with Canavan disease. Am J Med Genet 1988;29:463-71

13. Horska A, Kaufmann WE, Brant LJ, et al. In vivo quantitative proton MRS study of brain development from childhood to adolescence. J Magn Reson Imaging 2002;15:137-43

14. Kruse B, Barker PB, van Zijl PC, et al. Multislice proton magnetic resonance spectroscopic imaging in X-linked adrenoleukodystrophy. Ann Neurol 1994;36:595-608

15. Eichler FS, Itoh R, Barker PB, et al. Proton MR spectroscopic and diffusion tensor brain MR imaging in X-linked adrenoleukodystrophy: initial experience. Radiology 2002;225:245-52

16. Davie CA, Hawkins CP, Barker GJ, et al. Detection of myelin breakdown products by proton magnetic resonance spectroscopy. Lancet 1993;341:630-31

17. van der Voorn JP, Pouwels PJ, Hart AA, et al. Childhood white matter disorders: quantitative MR imaging and spectroscopy. Radiology 2006;241: $510-17$ 